\title{
IL-23 Expression in Stewart-Treves Syndrome: Two Case Reports and Immunohistochemical Investigation
}

\author{
Saaya Yoshida Taku Fujimura Kentaro Ohuchi Yumi Kambayashi \\ Yuichiro Segawa Emi Yamazaki Hisayuki Tono Toshiya Takahashi \\ Kenichiro Tsuchiyama Setsuya Aiba \\ Department of Dermatology, Tohoku University Graduate School of Medicine, Sendai, \\ Japan
}

Keywords

Angiogenesis · Angiosarcoma $\cdot$ IL-17 · IL-23 · Stewart-Treves syndrome

\begin{abstract}
Stewart-Treves syndrome (STS) is a rare cutaneous lymphangiosarcoma developing from chronic lymph edema as a consequence of radical mastectomy or surgical invasion of the groin for the treatment of cervical or penile cancer. Previous reports suggested possible mechanisms in the development of lymphangiosarcoma that correlate with the immunological background of STS patients. In this report, we described two cases of STS developing in patients who underwent radical dissection for cervical cancer, we employed immunohistochemical staining of IL-23 and IL-17.

\section{Introduction}

Stewart-Treves syndrome (STS) is a rare cutaneous lymphangiosarcoma developing from chronic lymph edema as a consequence of radical mastectomy or surgical invasion of the groin for the treatment of cervical or penile cancer [1]. STS develops not only in patients who have lymph edema secondary to radical resection of a tumor, but also in the form of congenital and acquired lymph edema (trauma, venous stasis, morbid obesity, etc.) [1]. Previous reports suggested possible mechanisms in the development of lymphangiosarcoma. Among them, Ruocco et al. [2] suggested that the development of STS correlates with immune suppression in immunocompromised hosts, suggesting that the host immunological background might correlate with the development of lymphangiosarcoma in STS. 


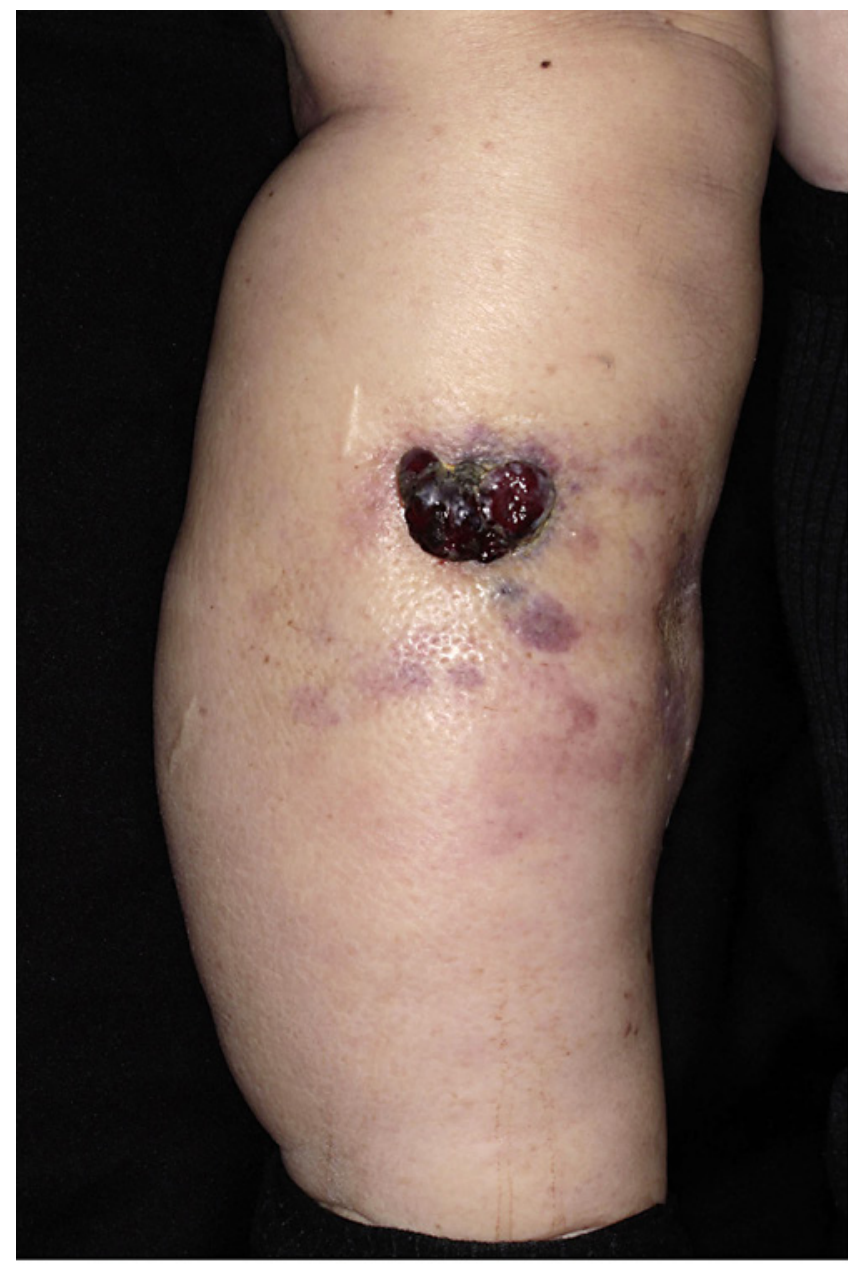

a

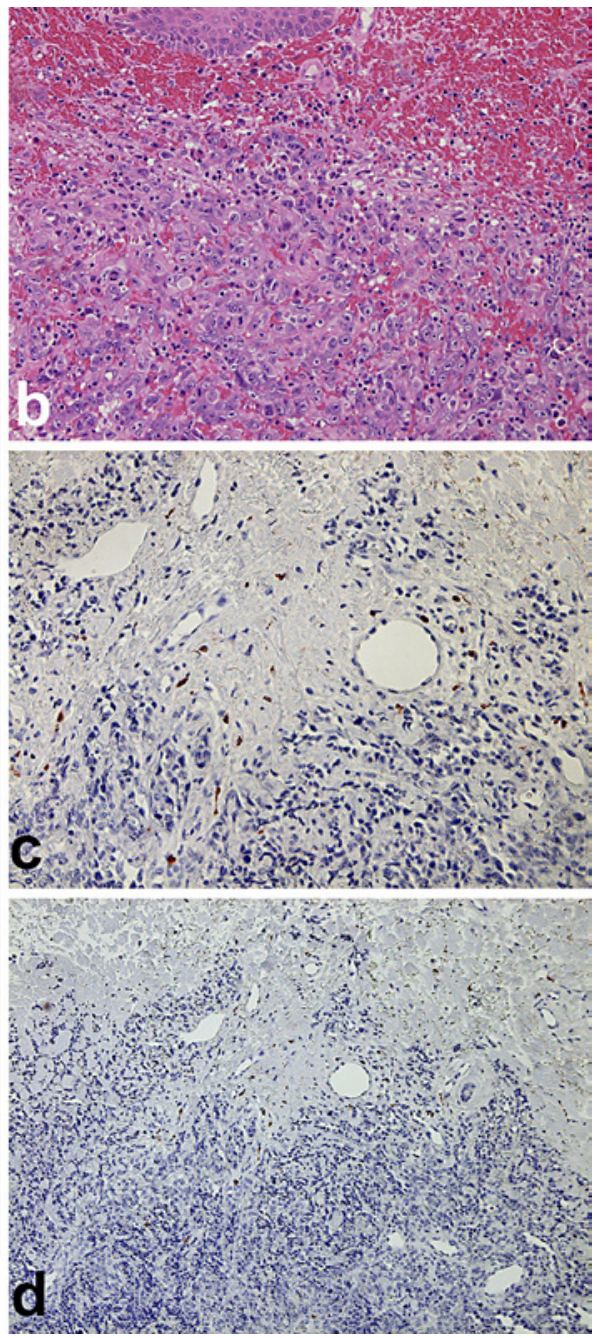

Fig. 1. A dark-red nodule with extended purpura on the right femur with prominent lymph edema (a). Irregularly anastomosing vascular channels lined by single layers of enlarged, atypical endothelial cells that existed between the collagen bundles (H\&E staining) (b). Immunohistochemical staining for case 1: IL-23 (c) and IL-17 (d).

\section{Case Report}

\section{Case 1}

A 79-year-old Japanese woman visited our outpatient clinic with a 1-month history of a red, easy to bleed, nodule on the right femur. She had undergone resection of a cervical cancer 24 years before and developed prominent lymph edema in the lower extremities. During her initial visit, physical examination revealed a dark-red nodule with extended purpura on the right femur together with prominent lymph edema (Fig. 1a). Histologically, these were irregularly anastomosing vascular channels lined by single layers of enlarged, atypical endothelial cells that existed between the collagen bundles (Fig. 1b). Immunohistochemical staining revealed that these atypical endothelial cells were positive for vimentin, CD31, CD34, D2-40, and Factor VIII. The Ki67 score was 90\%. Moreover, a substantial number of IL-23-producing cells (Fig. 1c) as well as IL-17-producing cells (Fig. 1d) were detected at the edge of the tumor mass. Positron emission tomography scans showed no evidence of metastases. From the 

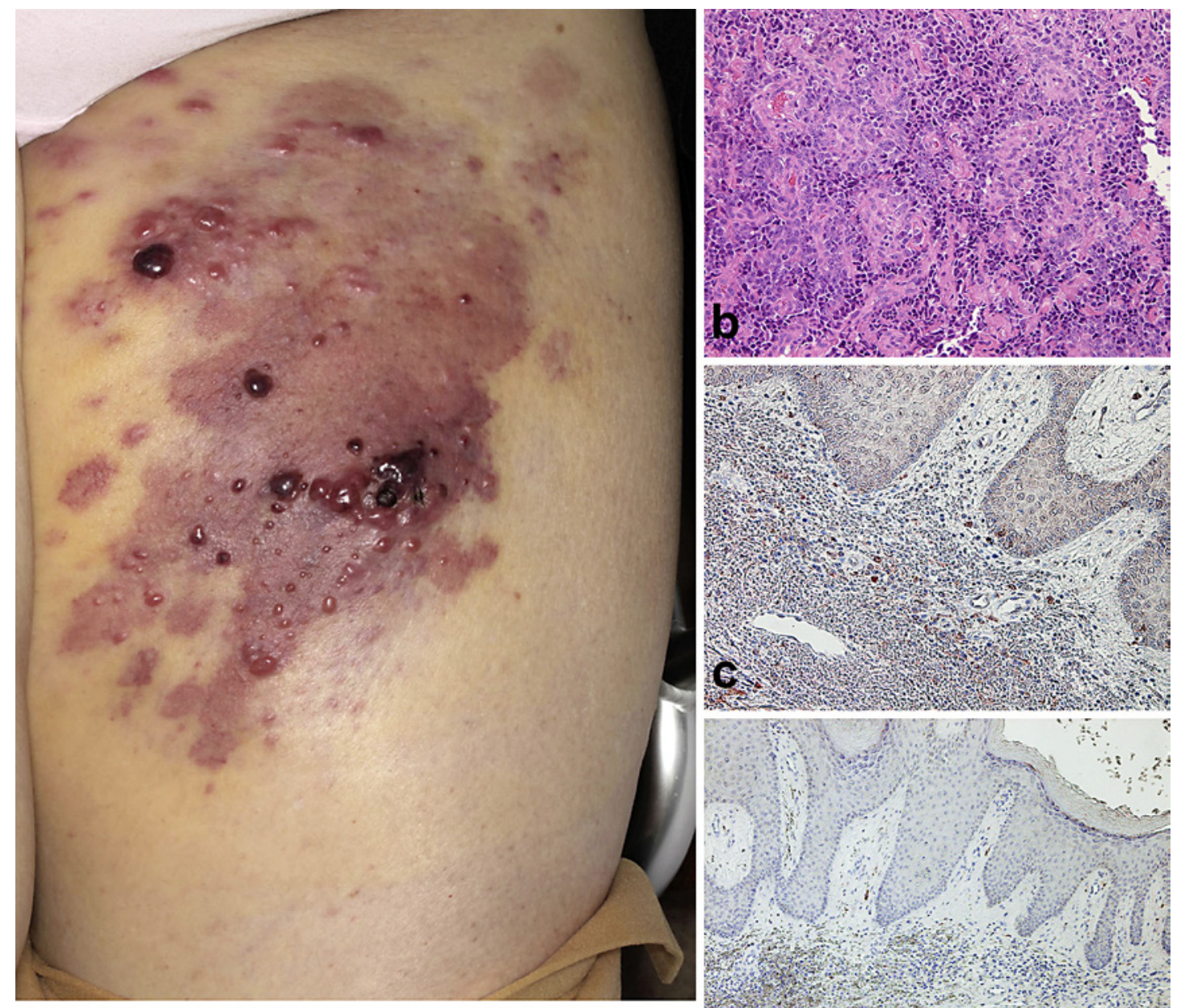

a

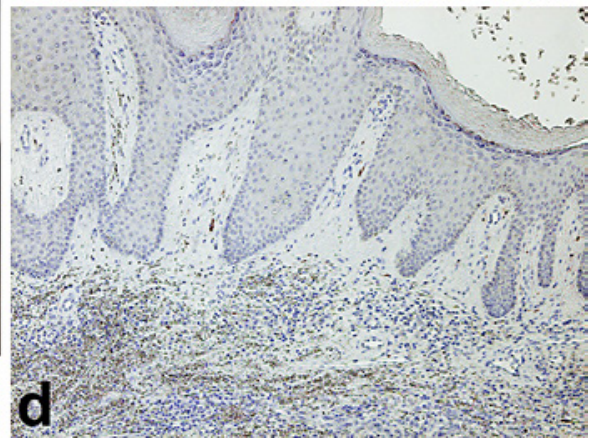

Fig. 2. Multiple dark-red nodules with extended purpura on the left femur with prominent lymph edema (a). Irregularly anastomosing vascular channels lined by single layers of enlarged, atypical endothelial cells that existed between collagen bundles with prominent apoptotic cells (b). Immunohistochemical staining for case 2: IL-23 (c) and IL-17 (d).

above findings, our diagnosis was STS. We administered radiation therapy (70 Gy in 35 fractions) to the femur along with docetaxel at a dose of $50 \mathrm{mg} / \mathrm{m}^{2}$ for a 4-week cycle with weekly oral administration of $17.5 \mathrm{mg}$ sodium risedronate hydrate. The tumor mass regressed rapidly, and the follow-up CT scan revealed no evidence of metastases 1 year after the administration of radiotherapy.

\section{Case 2}

A 72-year-old Japanese woman visited our outpatient clinic with a 5-month history of red, easy-to-bleed, nodules on her left femur. She had undergone resection of a cervical cancer, had been administered postoperative pelvicirradiation 18 years before, and developed prominent lymph edema in the lower extremities. During her initial visit, physical examination revealed multiple dark-red nodules with extended purpura on the left femur with prominent lymph edema (Fig. 2a). Histologically, these were irregularly anastomosing vascular channels lined by single layers of enlarged, atypical endothelial cells that existed 
between the collagen bundles with prominent apoptotic cells (Fig. 2b). Immunohistochemical staining revealed that these atypical endothelial cells were positive for vimentin, CD31, CD34, and D2-40. The Ki67 score was 90\%. Moreover, substantial numbers of IL-23-producing cells (Fig. 2c) as well as IL-17-producing cells (Fig. 2d) were detected at the edge of the tumor mass. CT scans showed no evidence of metastases. From the above findings, our diagnosis was STS. We administered radiation therapy (66 Gy in 33 fractions) to the femur along with docetaxel at a dose of $50 \mathrm{mg} / \mathrm{m}^{2}$ for a 4 -week cycle, but additional lesions developed around the irradiated area.

\section{Discussion}

IL-23 plays an important role in inducing Th17 cell proliferation as well as in the angiogenesis of tumors [3-7]. Indeed, Nie et al. [4] reported that IL-23 promotes the recruitment of M2 macrophages and neutrophils, which secrete immunosuppressive cytokines and vascular endothelial growth factor as well as matrix metalloproteinase 9 (MMP9) into tumor tissues. Since tumor-associated macrophages and tumor-associated neutrophils are significant components of the microenvironment of solid tumors in the majority of cancers $[8,9]$, IL-23 could be one of the crucial factors for the progression of cancers, including skin cancers. Indeed, as we have previously reported, Paget's cells as well as dermal myeloid cells produce IL-23, leading to the induction of IL-17 in the lesional skin in extramammary Paget's cells [7]. In addition to inducing IL-17 expression, IL-23 inhibits apoptosis; thus, promoting tumor progression [6]. In aggregate, IL-23 could be a key trigger for the induction of IL-17, leading to IL-17-producing cells in skin cancers.

IL-17-producing cells are widely detected and could play a significant role in skin cancers $[7,10-12]$. For example, several reports suggested the significance of IL-17 signaling in keratinocytes in the tumor formation of cutaneous squamous cell carcinoma [10-12]. More recently, we have also reported on the possible correlation between the CCL20/IL-23/IL-17 axis in the development of extramammary Paget's cells [7]. Notably, IL-17 is not only a signal for cell proliferation, but it also activates MMP2 and MMP9 to induce angiogenesis in several cancer types $[13,14]$. To understand the mechanisms for the induction of MMPs in skin, IL-17-related inflammatory models are important. Indeed, the significance of IL-17 was reported in autoimmune diseases such as psoriasis [15], bullous pemphigoid (BP) [16, 17], and alopecia areata [18]. Among them, Riani et al. [16] reported that IL-17 increased CXCL10 in inflammatory cells, leading to an augmented secretion of MMP9 from neutrophils and monocytes in BP patients. Notably, MMP9 remodels the extracellular matrix and promotes the sprouting and growth of new blood vessels [19] and, as we have previously reported, most cutaneous angiosarcomas produce MMP9 [20]. Since CXCL10 was reported as a predictive biomarker for cutaneous angiosarcoma [21], IL-17 might play a similar role in the tumor microenvironment of angiosarcoma-like BP.

In this report, we describe two cases of STS developing in patients who had previously undergone radical dissection of cervical cancer, for which we employed immunohistochemical staining of IL-23 and IL-17. Since we present only two cases, further cases are needed to prove that IL-23 and IL-17 are tumor-promoting factors in STS.

\section{Statement of Ethics}

The patients have given their written informed consent for the publication of their case including images. 


\section{Disclosure Statement}

The authors have no conflicting interests to declare.

\section{Funding Sources}

This study was supported in part by the Japan Agency for Medical Research and Development $(19 \mathrm{~cm} 0106434 \mathrm{~h} 0002)$.

\section{Author Contributions}

T. Fujimura designed the research study. S. Yoshida, T. Fujimura, Y. Kambayashi, Y. Segawa, E. Yamazaki, H. Tono, Y. Takahashi, and K. Tsuchiyama treated the patient and acquired the clinical data. K. Ohuchi and T. Fujimura wrote the manuscript. K. Ohuchi performed immunohistochemical staining. T. Fujimura and S. Aiba supervised the study.

\section{References}

1 Sharma A, Schwartz RA. Stewart-Treves syndrome: pathogenesis and management. J Am Acad Dermatol. 2012;67(6):1342-8.

2 Ruocco V, Ruocco E, Piccolo V, Brunetti G, Guerrera LP, Wolf R. The immunocompromised district in dermatology: A unifying pathogenic view of the regional immune dysregulation. Clin Dermatol. 2014;32(5):569-76.

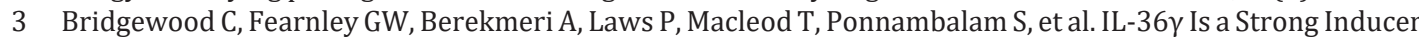
of IL-23 in Psoriatic Cells and Activates Angiogenesis. Front Immunol. 2018;9:200.

4 Nie W, Yu T, Sang Y, Gao X. Tumor-promoting effect of IL-23 in mammary cancer mediated by infiltration of M2 macrophages and neutrophils in tumor microenvironment. Biochem Biophys Res Commun. 2017;482(4): 1400-6.

5 Sutton CE, Lalor SJ, Sweeney CM, Brereton CF, Lavelle EC, Mills KH. Interleukin-1 and IL-23 induce innate IL-17 production from gammadelta T cells, amplifying Th17 responses and autoimmunity. Immunity. 2009;31(2): 331-41.

6 Sheng S, Zhang J, Ai J, Hao X, Luan R. Aberrant expression of IL-23/IL-23R in patients with breast cancer and its clinical significance. Mol Med Rep. 2018;17(3):4639-44.

7 Sato Y, Fujimura T, Tanita K, Chunbing L, Matsushita S, Fujisawa Y, et al. Malassezia-derived aryl hydrocarbon receptor ligands enhance the CCL20/Th17/soluble CD163 pathogenic axis in extra-mammary Paget's disease. Exp Dermatol. 2019;28(8):933-9.

8 Fujimura T, Kambayashi Y, Fujisawa Y, Hidaka T, Aiba S. Tumor-associated macrophages: Therapeutic targets for skin cancer. Front Oncol. 2018;8:3.

9 Masucci MT, Minopoli M, Carriero MV. Tumor Associated Neutrophils. Their Role in Tumorigenesis, Metastasis, Prognosis and Therapy. Front Oncol. 2019;9:1146.

10 Wu L, Chen X, Zhao J, Martin B, Zepp JA, Ko JS, et al. A novel IL-17 signaling pathway controlling keratinocyte proliferation and tumorigenesis via the TRAF4-ERK5 axis. J Exp Med. 2015:212(10):1571-87.

11 Nardinocchi L, Sonego G, Passarelli F, Avitabile S, Scarponi C, Failla CM, et al. Interleukin-17 and interleukin-22 promote tumor progression in human nonmelanoma skin cancer. Eur J Immunol. 2015;45(3):922-31.

12 Gasparoto TH, de Oliveira CE, de Freitas LT, Pinheiro CR, Ramos RN, da Silva AL, et al. Inflammatory events during murine squamous cell carcinoma development. J Inflamm (Lond). 2012;9(1):46.

13 Wu Z, He D, Zhao S, Wang H. IL-17A/IL-17RA promotes invasion and activates MMP-2 and MMP-9 expression via p38 MAPK signaling pathway in non-small cell lung cancer. Mol Cell Biochem. 2019;455(1-2):195-206.

14 Feng M, Wang Y, Chen K, Bian Z, J Jinfang Wu, Gao Q. IL-17A promotes the migration and invasiveness of cervical cancer cells by coordinately activating MMPs expression via the p38/NF- $\mathrm{kB}$ signal pathway. PLoS One. 2014;9(9):e108502.

15 Soderstrom C, Berstein G, Zhang W, Valdez H, Fitz L, Kuhn M, et al. Ultra-Sensitive Measurement of IL-17A and IL-17F in Psoriasis Patient Serum and Skin. AAPS J. 2017;19(4):1218-22.

16 Riani M, Le Jan S, Plée J, Durlach A, Le Naour R, Haegeman G, et al. Bullous pemphigoid outcome is associated with CXCL10-induced matrix metalloproteinase 9 secretion from monocytes and neutrophils but not lymphocytes. J Allergy Clin Immunol. 2017;139(3):863-e3. 
17 Furudate S, Fujimura T, Kambayashi Y, Kakizaki A, Aiba S. Comparison of CD163+ CD206+ M2 macrophages in the lesional skin of bullous pemphigoid and pemphigus vulgaris: the possible pathogenesis of bullous pemphigoid. Dermatology. 2014;229(4):369-78.

18 Tojo G, Fujimura T, Kawano M, Ogasawara K, Kambayashi Y, Furudate S, et al. Comparison of interleukin-17producing cells in different clinical types of alopecia areata. Dermatology (Basel). 2013;227(1):78-82.

19 Heissig B, Hattori K, Dias S, Friedrich M, Ferris B, Hackett NR, et al. Recruitment of Stem and Progenitor Cells from the Bone Marrow Niche Requires MMP-9 Mediated Release of Kit-Ligand. Cell. 2002;109(5):625-37.

20 Ishibashi M, Fujimura T, Hashimoto A, Haga T, Onami K, Tsukada A, et al. Successful Treatment of MMP9-Expressing Angiosarcoma with Low-Dose Docetaxel and Bisphosphonate. Case Rep Dermatol. 2012;4(1): 5-9.

21 Fujimura T, Sato Y, Kambayashi Y, Tanita K, Tsukada A, Terui H, et al. Three patients with advanced cutaneous angiosarcoma treated with eribulin: investigation of serum soluble CD163 and chemokine (C-X-C motif) ligand 10 as possible biomarkers predicting the biological behaviour of angiosarcoma. Br J Dermatol. 2018; 179(6):1392-5. 